To appear in the Astrophysical Journal

\title{
XMM-Newton X-ray Observations of LkCa 15: A T Tauri Star With a Formative Planetary System
}

\author{
Stephen L. Skinner ${ }^{1}$ and Manuel Güdel ${ }^{2}$
}

\begin{abstract}
High-resolution ground-based images of the T Tauri star LkCa 15 have revealed multiple companions that are thought to comprise a formative planetary system. The candidate protoplanets orbit at distances $\sim 15-20$ AU within the dust-depleted inner region of the circumstellar disk. Because of its young age ( 1 - 4 Myr), LkCa 15 provides a benchmark system for testing planet-formation models. We detected LkCa 15 as a bright X-ray source in a short $10 \mathrm{ks}$ Chandra observation in 2009. We report here new results obtained from a deeper $37 \mathrm{ks}$ $X M M$-Newton observation in 2014. The new data provide better sampling in the time domain and improved sensitivity at low energies below $1 \mathrm{keV}$. Spectral fits with thermal emission models require at least two temperature components at $\mathrm{kT}_{\text {cool }} \approx 0.4 \mathrm{keV}$ and $\mathrm{kT}_{\text {hot }} \approx 2.2 \mathrm{keV}$. The value of $\mathrm{kT}_{\text {hot }}$ is about a factor of two less than inferred from Chandra, suggesting that the hot-component temperature is variable. The best-fit absorption column density is in good agreement with that expected from optical extinction estimates $A_{V} \approx 1.3-1.7$ mag. The intrinsic $\mathrm{X}$ ray luminosity is $\mathrm{L}_{x}(0.2-10 \mathrm{keV})=3 \times 10^{30} \mathrm{ergs} \mathrm{s}^{-1}$. Estimates of the X-ray heating rate of the inner disk and protoplanets are sensitive to the assumed disk gas surface density for which recent ALMA observations give estimates $\Sigma_{0, \text { gas }} \sim$ $10^{2} \mathrm{~g} \mathrm{~cm}^{-2}$ at $1 \mathrm{AU}$ from the star. At such densities, X-ray heating is confined mainly to the upper disk layers and $\mathrm{X}$-ray penetration through the disk midplane to the protoplanets at $r \approx 15-20 \mathrm{AU}$ is negligible.
\end{abstract}

Subject headings: stars: individual (LkCa 15; NSVS 6777197) — accretion, accretion disks — stars: pre-main sequence — X-rays: stars

\footnotetext{
${ }^{1}$ CASA, Univ. of Colorado, Boulder, CO, USA 80309-0389; stephen.skinner@colorado.edu

${ }^{2}$ Dept. of Astronomy, Univ. of Vienna, Türkenschanzstr. 17, A-1180 Vienna, Austria; manuel.guedel@univie.ac.at
} 


\section{Introduction}

Although several thousand exoplanets have now been discovered 3 , examples of exoplanets orbiting young pre-main sequence (PMS) stars are rare. The identification of planethosting young stars of ages a few Myr and study of their circumstellar disks provide valuable insight into the circumstellar environment in which planets (and planetary systems) form and how the planets affect disk properties such as gas and dust distribution. As such, observational studies of disks and exoplanets around PMS host stars provide crucial constraints on planet-formation models.

Perhaps the most striking example to emerge so far of a PMS star hosting a protoplanet, and possibly even a protoplanetary system, is the accreting classical T Tauri star (cTTS) LkCa 15 in the Taurus star-forming region (Table 1). In a remarkable discovery, Kraus \& Ireland (2012, hereafter KI12) reported the direct detection using infrared masked aperture interferometry of a suspected protoplanet at a projected separation of $71.9 \pm 1.6$ mas from $\mathrm{LkCa}$ 15. The protoplanet is located in the dust-depleted inner region of the LkCa 15 disk at a deprojected orbital radius of $\sim 16$ - 20 AU. Further monitoring in the near-IR has detected possible orbital motion of the protoplanet (Ireland \& Kraus 2014).

Extensive and ongoing studies of the disk surrounding the host star LkCa 15 at infrared, (sub)millimeter, and radio wavelengths show that it is severely depleted of dust inside a radius of $\sim 45$ - 50 AU (Andrews et al 2011a,b - hereafter A11a,b; Isella et al. 2012, 2014; Espaillat et al. 2008, 2010; Thalmann et al. 2014, 2016). Although the inner region is dust-depleted, there is still gas present as revealed by ${ }^{12} \mathrm{CO}$ and ${ }^{13} \mathrm{CO}$ observations (Piétu et al. 2007; van der Marel et al. 2015). Also, Isella et al. (2014) detected a compact 7 mm continuum source with the Very Large Array (VLA) at the position of the central star. They conclude that the $7 \mathrm{~mm}$ emission is not consistent with a stellar photospheric origin but could be due to either millimeter size grains near the star or ionized gas in the vicinity of the star. Plausible explanations for the dust-clearing in the inner disk are variable dust grain sizes and opacity (Isella et al. 2012) or dynamical clearing by one or more orbiting objects (A11a,b), with the latter explanation currently favored.

The possibility that more than one protoplanet might be present has received some recent support from new high-contrast near-IR imaging and adaptive optics $\mathrm{H} \alpha$ imaging of LkCa 15 obtained by Sallum et al. (2015). They report the detection of three distinct objects with best-fit orbital semi-major axes in the range 14.7 - 18.6 AU. One of these objects is

\footnotetext{
${ }^{3}$ For a current catalog of exoplanets, see the NASA Exoplanet Archive database at http://http://exoplanetarchive.ipac.caltech.edu
} 
the suspected protoplanet that was designated as LkCa $15 \mathrm{~b}$ by KI12 but is referred to as LkCa 15c by Sallum et al. (2015). The latter authors obtain a semi-major axis $a=18.6$ $(+2.5,-2.7) \mathrm{AU}$ for this object. They also report the direct near-IR and $\mathrm{H} \alpha$ detection of an object orbiting closer to the star at $a=14.7( \pm 2.1)$ AU which they characterize as an accreting protoplanet. A third fainter object at $a \sim 18 \mathrm{AU}$ was detected at L'-band only and its properties are not yet well-constrained. Studies of the inner disk and candidate protoplanets are ongoing. High spatial resolution scattered light images of the inner disk of LkCa 15 based on J-band imaging polarimetry have now been obtained by Thalmann et al. (2016). Their images show structure from scattering material at the positions of the candidate protoplanets which they argue could be responsible for some of the signal reported in previous aperture-masking observations.

The likely presence of a formative planetary system around LkCa 15 provides an unprecedented opportunity to study the early stages of planet formation in detail at high effective spatial resolution given the modest distance of $\sim 140$ pc to the Taurus star-forming region. Our study presented here focuses on the effects of X-ray irradiation by the central star on the gas-dominated inner disk. We detected LkCa 15 as a bright X-ray source in a previous Chandra observation and provided initial estimates of the X-ray ionization and heating rates in the inner disk (Skinner \& Güdel 2013, hereafter SG13).

We report here the results of a more recent X-ray observation of LkCa 15 obtained with XMM-Newton. These observations provide better sensitivity at low energies below 1 $\mathrm{keV}$ where X-ray absorption due to intervening gas becomes important. The improved lowenergy sensitivity provides tighter constraints on the absorption toward the star as measured by the equivalent neutral hydrogen column density $\mathrm{N}_{\mathrm{H}}$, and the intrinsic (unabsorbed) X-ray luminosity $\left(\mathrm{L}_{x}\right)$. Fits of the XMM-Newton spectra confirm the earlier Chandra result that cool and hot plasma components are present but the temperature of the hot component is lower than was measured with Chandra and variability of the hotter plasma seems likely. $\mathrm{X}$-ray ionization and heating rates of the inner disk are recomputed based on the new XMMNewton results and revised inner disk model parameters from recent ALMA observations.

The importance of the effects of X-ray and extreme-ultraviolet (EUV) radiation on disks and protoplanetary systems around young stars has been noted in several previous studies of which two examples are Igea \& Glassgold (1999; hereafter IG99) and CecchiPestellini, Ciaravella, \& Micela (2006). X-ray and EUV emission ionizes and heats disk gas (especially in the outer surface layers), affects disk chemistry, accretion, and mass-loss (via photoevaporation), and strengthens the coupling between the accretion disk and the stellar magnetic field. Since X-rays influence mass-loss and disk dissipation they are one of the factors that constrain the timescale for planet formation. 
Table 1. Properties of LkCa 15

\begin{tabular}{lll}
\hline \hline \multicolumn{1}{c}{ Property } & \multicolumn{1}{c}{ Value } & Refs. \\
\hline Sp. type & $\mathrm{K} 5 \pm 2$ & 1,2 \\
Age $(\mathrm{Myr})$ & $2[1-4]$ & 3 \\
$\mathrm{M}_{*}\left(\mathrm{M}_{\odot}\right)$ & 1.0 & 4 \\
$\mathrm{R}_{*}\left(\mathrm{R}_{\odot}\right)$ & 1.6 & 2 \\
$\mathrm{~T}_{\text {eff }}(\mathrm{K})$ & 4730 & 1 \\
$\mathrm{~A}_{\mathrm{V}}(\mathrm{mag})$ & $0.62 ; 1.3-1.7$ & 1,2 \\
$i_{\text {disk }}(\mathrm{deg})$. & $50.5-52$ & 5 \\
$\mathrm{M}_{\text {disk }}\left(\mathrm{M}_{\odot}\right)$ & $\approx 0.1$ & 5,6 \\
$\mathrm{~d}(\mathrm{pc})$ & 140 & 7 \\
$\mathrm{~L}_{*}\left(\mathrm{~L}_{\odot}\right)$ & $0.74-1.2$ & 4,8 \\
$\log \mathrm{L}_{x}\left(\operatorname{ergs~s}^{-1}\right)$ & $30.47[30.40-30.52]$ & 9,10 \\
\hline
\end{tabular}

Note. - Refs. (1) Kenyon \& Hartmann 1995 (2) Espaillat et al. 2010 (3) Kraus \& Hillenbrand 2009 (4) Simon et al. 2000 (5) Isella et al. 2012 (6) van der Marel et al. 2015 (7) Torres et al. 2009 (8) Andrews et al. 2011b (9) Skinner \& Güdel 2013 (10) this work 


\section{Previous Chandra Observation}

We obtained a 9.8 ks Chandra observation (ObsId 10999) of LkCa 15 on 27 December 2009 using the ACIS-S (Advanced CCD Imaging Spectrometer) array. Results were presented by SG13 and are briefly summarized here. LkCa 15 was detected with 590 net counts (0.2 - 8 $\mathrm{keV}$ ). No statistically significant variability was present in the X-ray light curve. Acceptable spectral fits were obtained using a two-temperature (2T) apec thermal plasma model and abundances typical of TTS in Taurus. The best-fit model gave an absorption column density $\mathrm{N}_{\mathrm{H}}=3.7[2.4-5.1] \times 10^{21} \mathrm{~cm}^{-2}$ and plasma temperatures $\mathrm{kT}_{\text {cool }}=0.30[0.25-0.37 \mathrm{keV}]$, $\mathrm{kT}_{\text {hot }}=5.1[3.0-13.6] \mathrm{keV}$, where brackets enclose $90 \%$ confidence intervals. The intrinsic (unabsorbed) luminosity was $\log \mathrm{L}_{x}(0.3-10 \mathrm{keV})=30.4 \mathrm{ergs} \mathrm{s}^{-1}$ at an assumed distance of 140 pc.

\section{XMM-Newton Observation}

The XMM-Newton observation (ObsId 0722340101) began on 20 February 2014 at 19:12 UTC and ended on 21 February at 06:02 UTC. Data were acquired with the European Photon Imaging Camera (EPIC) in Full-Frame mode using the Medium optical blocking filter. EPIC provides charge-coupled device (CCD) imaging spectroscopy from the pn camera (Strüder et al. 2001) and two nearly identical MOS cameras (MOS1 and MOS2; Turner et al. 2001). The EPIC cameras have energy coverage in the range $\mathrm{E} \approx 0.2-15 \mathrm{keV}$ with energy resolution $\mathrm{E} / \Delta \mathrm{E} \approx 20-50$. The MOS cameras provide the best on-axis angular resolution with FWHM $\approx 4.3^{\prime \prime}$ at $1.5 \mathrm{keV}$.

Data were reduced with the XMM-Newton Science Analysis System (SAS vers. 15.0) using standard procedures including the filtering of raw event data to select good event patterns and removal of data within time intervals of high background radiation. The usable exposures obtained after removing high background intervals and total exposures (in parentheses) were 27.8 (36.04) ks for pn, 35.8 (37.70) ks for MOS1, and 36.6 (37.67) ks for MOS2. Thus, about $22 \%$ of the pn exposure was adversely affected by high background but the MOS exposures were not severely affected.

A circular region centered on $\mathrm{LkCa} 15$ with a radius $\mathrm{r}=20^{\prime \prime}(\approx 80 \%$ encircled energy at $1.5 \mathrm{keV}$ ) was used to extract X-ray light curves and spectra. Background analysis was conducted on circular source-free regions near the source. The SAS tasks rmfgen and arfgen were used to generate source-specific RMFs and ARFs for spectral analysis. The data were analyzed using the HEASOFT Xanadu software package. 


\section{Results}

Figure 1 shows the EPIC pn image of LkCa 15 and its surroundings. LkCa 15 is prominently detected at pn centroid position R.A. $=04^{h} 39^{m} 17^{s} .79$, decl. $=+22^{\circ} 21^{\prime}$ $03^{\prime \prime} .08$ (J2000). The previous Chandra observation had somewhat better spatial resolution and gave an X-ray position R.A. $=04^{h} 39^{m} 17^{s} .787$, decl. $=+22^{\circ} 21^{\prime} 03^{\prime \prime} .28$ (SG13). These X-ray positions are in good agreement with the HST GSC v2.3 position R.A. $=04^{h} 39^{m}$ $17^{s} .787$, decl. $=+22^{\circ} 21^{\prime} 03^{\prime \prime} .26$. Evidence for binarity in LkCa 15 has so far not been found (Nguyen et al. 2012).

There are no other X-ray sources in the immediate vicinity of LkCa 15 . The nearest EPIC source detected by the pipeline processing software lies more than $1^{\prime}$ from LkCa 15. The K5 star HD 284589 located 27."6 north of LkCa 15 is not detected, nor was it detected by Chandra. The only other bright stellar X-ray source in the EPIC field-of-view is the eclipsing Algol-type binary system NSVS 6777197 (2MASS J04394628+2211503) located $\approx 11^{\prime}$ southeast of LkCa 15 (Fig. 1). Its X-ray properties are summarized further below.

The EPIC light curves of LkCa 15 are shown in Figure 2. No large-amplitude fluctuations or flares are present but there is a slow falloff in the count rate during the observation. Checks for variability on binned background-subtracted broad-band $(0.2-8 \mathrm{keV})$ light curves using the $\chi^{2}$ test give a high variability probability. Using $1000 \mathrm{~s}$ bins, the probability of variability is $\mathrm{P}_{\text {var }}(0.2-8 \mathrm{keV})=0.96(\mathrm{pn}), 0.99$ (MOS1), and 0.98 (MOS2).

Figure 3 compares the EPIC pn spectrum with the previous Chandra ACIS-S spectrum and shows an overlay of the two EPIC MOS spectra. A notable difference is that there is no significant emission below $\approx 0.5 \mathrm{keV}$ in the ACIS-S spectrum but the higher EPIC pn effective area at low energies reveals detectable emission down to $\approx 0.2 \mathrm{keV}$. This leads to a more reliable determination of $\mathrm{N}_{\mathrm{H}}$ from the EPIC pn spectrum since lower-energy photons are more susceptible to absorption. The only line feature clearly visible is the Ne X Ly $\alpha$ line at $1.02 \mathrm{keV}$ which is seen in both MOS spectra. There is also a weak feature at $1.86 \mathrm{keV}$ in the pn spectrum that may be Si XIII.

Spectra were fitted using the Astrophysical Plasma Emission Code variable-abundance vapec model in XSPEC version 12.8.2 (Smith et al. 2001). Photoelectric absorption was applied using the XSPEC wabs model to determine the equivalent hydrogen column density $\left(\mathrm{N}_{\mathrm{H}}\right)$. A two-temperature (2T) vapec model was required to obtain acceptable fits. A comparison of fit results for two vapec models using different abundances is given in Table 2. Significant improvement relative to the solar-abundance fit is obtained by allowing the abundances of $\mathrm{Ne}$ and Fe to vary (model A in Table 2). Very little further improvement in the fit is obtained by letting the abundances of other metals to deviate from solar values. 
Model B uses typical TTS abundances for the Taurus Molecular Cloud (Güdel et al. 2007; Scelsi et al. 2007), as were adopted in the Chandra analysis (Model C of SG13). The Ne abundance inferred from Model $\mathrm{A}$ is $\mathrm{Ne}=2.4$ [2.07 - 2.71] times solar, but the generic Taurus abundances (Model B) keep the value fixed at $\mathrm{Ne}=0.83$ times solar.

The EPIC fit results for Models $\mathrm{A}$ and $\mathrm{B}$ are overall quite similar. Cool plasma at $\mathrm{kT}_{1}$ $\approx 0.4 \mathrm{keV}$ is required by both models, consistent with the value obtained from previous Chandra ACIS-S data (SG13). A hotter component at $\mathrm{kT}_{2} \approx 2.1-2.4 \mathrm{keV}$ is also required. This temperature is about a factor of two lower than was inferred from Chandra, which gave $\mathrm{kT}_{2} \approx 4-5 \mathrm{keV}$. The $90 \%$ confidence levels from EPIC are $\mathrm{kT}_{2}=[1.93-2.40] \mathrm{keV}$ (model $\mathrm{B})$, which does not overlap the corresponding ACIS-S range $\mathrm{kT}_{2}=[3.0-13.6] \mathrm{keV}$. This suggests that the temperature of the hot component is variable. Model B associates a larger percentage of the volume emission measure with the cool plasma component, as determined by the XSPEC normalization parameter (norm).

The best-fit absorption column density is consistent with that expected from $A_{V}=1.3$ - 1.7 (Table 1; Espaillat et al. 2010). Using the $\mathrm{N}_{\mathrm{H}}$ to $\mathrm{A}_{\mathrm{V}}$ conversion of Vuong et al. (2003), model A gives $\mathrm{A}_{\mathrm{V}}=1.4$ [1.3 - 1.6] and model $\mathrm{B}$ yields $\mathrm{A}_{\mathrm{V}}=1.8$ [1.7 - 1.9]. The Gorenstein (1975) coversion gives $A_{V}$ values that are about $25 \%$ lower than above. Thus, there is no convincing evidence for excess X-ray absorption above that expected from $A_{V}$. The ratio of $\mathrm{X}$-ray to stellar luminosity from the EPIC fits is $\log \mathrm{L}_{x}(0.2-10 \mathrm{keV}) / \mathrm{L}_{*}=-3.16$ to -3.06 . This ratio is in good agreement with other TTS in Taurus as determined by Telleschi et al. (2007).

NSVS 677719\%: The star NSVS 6777197 was serendipitously detected as a bright X-ray source by EPIC pn (Fig. 1) and MOS2, but was outside the MOS1 field-of-view. It was classified as a detached Algol binary with a 3.928 d period by Drake et al. (2014). Algoltype binaries usually have one component of late spectral type and are often detected as bright coronal X-ray sources (Singh, Drake, \& White 1996). Large X-ray flares can occur as seen in the prototype Algol (Schmitt \& Favata 1999). But the EPIC X-ray light curves of NSVS 6777197 show no significant variability. Simultaneous spectral fits of the pn and MOS2 spectra with a $2 \mathrm{~T}$ vapec thermal plasma model give an absorption column density $\mathrm{N}_{\mathrm{H}}$ $=1.78[1.57-2.04] \times 10^{21} \mathrm{~cm}^{-2}, \mathrm{kT}_{\text {cool }}=0.92[0.79-1.04] \mathrm{keV}, \mathrm{kT}_{\text {hot }}=2.60[2.23-3.26]$ $\mathrm{keV}$, and iron abundance $\mathrm{Fe}=0.22[0.10-0.36]$ solar, where brackets enclose $90 \%$ confidence intervals. The absorbed (and unabsorbed) fluxes are $\mathrm{F}_{x}(0.2-10 \mathrm{keV})=7.2 \times 10^{-13}(1.10 \times$ $\left.10^{-12}\right)$ ergs $\mathrm{cm}^{-2} \mathrm{~s}^{-1}$. 
Table 2. XMM-Newton Spectral Fits for LkCa 15

\begin{tabular}{|c|c|c|}
\hline \multicolumn{3}{|l|}{ Parameter } \\
\hline Model & $\mathrm{A}$ & $\mathrm{B}$ \\
\hline Emission $^{\mathrm{a}}$ & Thermal $(2 \mathrm{~T})$ & Thermal (2T) \\
\hline Abundances & non-solarc & non-solard \\
\hline $\mathrm{N}_{\mathrm{H}}\left(10^{22} \mathrm{~cm}^{-2}\right)$ & $0.23[0.21-0.25]$ & $0.29[0.27-0.31]$ \\
\hline $\mathrm{kT}_{1}(\mathrm{keV})$ & $0.41[0.39-0.44]$ & $0.40[0.39-0.42]$ \\
\hline $\mathrm{kT}_{2}(\mathrm{keV})$ & $2.39[2.20-2.59]$ & $2.08[1.93-2.40]$ \\
\hline norm $_{1}\left(10^{-4}\right)^{b}$ & $2.75[2.31-3.29]$ & $8.26[7.33-9.28]$ \\
\hline norm $_{2}\left(10^{-4}\right)^{\mathrm{b}}$ & $3.06[2.91-3.21]$ & $4.22[3.84-4.50]$ \\
\hline$\chi^{2} /$ dof & $862.0 / 634$ & $850.4 / 636$ \\
\hline$\chi_{\text {red }}^{2}$ & 1.36 & 1.34 \\
\hline $\mathrm{F}_{\mathrm{X}}\left(10^{-12} \mathrm{ergs} \mathrm{cm}^{-2} \mathrm{~s}^{-1}\right)$ & $0.55(1.13)$ & $0.54(1.43)$ \\
\hline $\mathrm{F}_{\mathrm{X}, 1}\left(10^{-12} \operatorname{ergs~cm}^{-2} \mathrm{~s}^{-1}\right)$ & $0.23(0.64)$ & $0.24(0.92)$ \\
\hline $\mathrm{F}_{\mathrm{X}, 2}\left(10^{-12} \mathrm{ergs} \mathrm{cm}^{-2} \mathrm{~s}^{-1}\right)$ & $0.32(0.49)$ & $0.29(0.51)$ \\
\hline $\log L_{X}\left(\operatorname{ergs~s}^{-1}\right)$ & 30.42 & 30.52 \\
\hline $\log L_{X, 1}\left(\operatorname{ergs~s}^{-1}\right)$ & 30.18 & 30.33 \\
\hline $\log \mathrm{L}_{\mathrm{X}, 2}\left(\operatorname{ergs~s}^{-1}\right)$ & 30.06 & 30.08 \\
\hline $\log \left[\mathrm{L}_{\mathrm{X}} / \mathrm{L}_{*}\right]$ & -3.16 & -3.06 \\
\hline
\end{tabular}

Note. - Based on XSPEC (version 12.8.2) simultaneous fits of the background-subtracted EPIC spectra (pn, MOS1, MOS2) binned to a minimum of 10 counts per bin with high background time intervals removed. The fits were obtained with $2 \mathrm{~T}$ vapec optically plasma models. The tabulated parameters are absorption column density $\left(\mathrm{N}_{\mathrm{H}}\right)$, plasma energy (kT), and XSPEC component normalization (norm). Abundances are referenced to the solar values of Anders \& Grevesse (1989). Square brackets enclose $90 \%$ confidence intervals. The total X-ray flux $\left(\mathrm{F}_{\mathrm{X}}\right)$ and fluxes associated with each model component $\left(\mathrm{F}_{\mathrm{X}, \mathrm{i}}\right)$ are the absorbed values in the $0.2-10 \mathrm{keV}$ range, followed in parentheses by unabsorbed values. The total X-ray luminosity $\mathrm{L}_{\mathrm{X}}$ and luminosities of each component $\mathrm{L}_{\mathrm{X}, \mathrm{i}}$ are unabsorbed values in the $0.2-10 \mathrm{keV}$ range and assume a distance of 140 pc. A value $\mathrm{L}_{*}=1.0 \mathrm{~L}_{\odot}$ is adopted based on an average of values given in the literature.

${ }^{\text {a }}$ Models $\mathrm{A}$ and $\mathrm{B}$ are of form: $\mathrm{N}_{\mathrm{H}} \cdot\left(\mathrm{kT}_{1}+\mathrm{kT}_{2}\right)$

${ }^{\mathrm{b}}$ For thermal apec models, the norm is related to the volume emission measure $\left(\mathrm{EM}=\mathrm{n}_{e}^{2} \mathrm{~V}\right)$ by $\mathrm{EM}=4 \pi 10^{14} \mathrm{~d}_{c m}^{2} \times$ norm, where $\mathrm{d}_{c m}$ is the stellar distance in $\mathrm{cm}$. At $\mathrm{d}=140 \mathrm{pc}$ this becomes $\mathrm{EM}=2.34 \times 10^{56} \times$ norm $\left(\mathrm{cm}^{-3}\right)$.

${ }^{\mathrm{c}}$ All abundances were held fixed at their solar values except for $\mathrm{Ne}$ and $\mathrm{Fe}$ which were allowed to vary and converged to $\mathrm{Ne}=2.37[2.07-$ $2.71]$ and $\mathrm{Fe}=0.45[0.39-0.53]$ relative to their solar values.

dAbundances were held fixed at typical values for TTS in Taurus (Güdel et al. 2007; Scelsi et al. 2007). These are (relative to solar): H $=1.0, \mathrm{He}=1.0, \mathrm{C}=0.45, \mathrm{~N}=0.79, \mathrm{O}=0.43, \mathrm{Ne}=0.83, \mathrm{Mg}=0.26$, $\mathrm{Al}=0.50, \mathrm{Si}=0.31, \mathrm{~S}=0.42, \mathrm{Ar}=0.55, \mathrm{Ca}=0.195, \mathrm{Fe}=0.195, \mathrm{Ni}$ $=0.195$. 


\section{Discussion}

\subsection{X-ray Heating and Ionization}

Disk X-ray heating is due to fast electrons ejected by atoms during X-ray ionization. A complete discussion of the methodology for computing the X-ray ionization rates $(\zeta)$ and heating rates $\left(\Gamma_{x}\right)$ in the disk can be found in the Chandra study of LkCa 15 (SG13) and the references cited below. We have recomputed the disk ionization and heating rates for LkCa 15 based on plasma temperature $\left(\mathrm{kT}_{x}\right)$ and X-ray luminosity $\left(\mathrm{L}_{x}\right)$ values determined from the XMM-Newton spectral fits (Table 2). The revised rates for the cool and hot plasma components are summarized in Table 3.

\subsubsection{Disk Model}

A disk model must be adopted in order to compute X-ray ionization and heating rates. A key disk parameter is the density of H-nuclei $\left(n_{H}\right)$, which is required to compute the X-ray absorption and heating rate per unit volume at a given position in the disk. The value of $n_{H}$ is determined by the disk gas surface density $\Sigma$ which is not yet well-constrained observationally in the inner disk because of limitations on telescope angular resolution, especially at (sub)mm wavelengths.

A cylindrical coordinate system is used to specify positions $(r, z)$ in the disk where the radial coordinate $r$ is the distance in the midplane from the center of the star to the specified point and $z$ is the height above the disk midplane. Azimuthal symmetry is assumed. We adopt a radial disk temperature profile of the form $\mathrm{T}(r)=400(r / 1 \mathrm{AU})^{-0.5} \mathrm{~K}$ based on a stellar effective temperature $\mathrm{T}_{\text {eff }}=4730 \mathrm{~K}$. Vertical temperature gradients are ignored (IG99). For the above temperature relation and adopted stellar parameters (Table 1) the disk scale height at $r=1 \mathrm{AU}$ is $\mathrm{H}_{0} \equiv \mathrm{H}(r=1 \mathrm{AU})=7.1 \times 10^{11} \mathrm{~cm}$ and scales as $\mathrm{H}(r) \propto$ $r^{+1.25}$.

We adopt a simple power-law for the gas surface density $\Sigma(r)=\Sigma_{0}(r / 1 \mathrm{AU})^{q}$, normalized to $\Sigma_{0}=10^{2} \mathrm{~g} \mathrm{~cm}^{-2}$ and a power-law exponent $q=-1$. This simple power-law ignores the additional exponential decay term included in the surface density profiles of A11b and van der Marel et al. (2015) since its value is near unity for the inner disk radii $r \lesssim 15$ AU of interest here. The above value of $\Sigma_{0}$ is comparable to that determined from the recent studies of Manara et al. (2014) and van der Marel et al. (2015) but must be interpreted as an order-of-magnitude estimate due to several factors that limit our knowledge of the gas distribution in the spatially-unresolved inner disk (Sec. 5.2). We also note that the value of 
$\Sigma_{0}$ adopted here is much higher than the value $\Sigma_{0}=10^{-3} \mathrm{~g} \mathrm{~cm}^{-2}$ used in SG13 that was based on the earlier LkCa 15 disk study of A11b (Sec. 5.2).

We adopt the abundance ratio $\mathrm{He} / \mathrm{H}=0.1$ by number and assume that hydrogen in the disk is predominantly molecular. The mass density at the midplane is $\rho(r, z=0)=$ $0.4 \Sigma(r) / \mathrm{H}(r)$ which at $r=1$ AU gives $\rho_{0} \equiv \rho(r=1 \mathrm{AU}, z=0)=5.63 \times 10^{-16} \mathrm{~g} \mathrm{~cm}^{-3}$. The number density of $\mathrm{H}$-nuclei is given by $\mathrm{n}_{\mathrm{H}}=\rho /\left(\mu \mathrm{m}_{p}\right)$ where $\mathrm{m}_{p}$ is the proton mass and $\mu=$ 1.42 for H-nuclei (Glassgold et al. 2004).

\subsubsection{Disk Ionization and Heating}

Calculation of the X-ray ionization and heating rates in the disk is based on the analytic results of Glassgold et al. (1997a, hereafter G97a; Glassgold et al. 1997b), IG99, Shang et al. (2002; hereafter S02), and Glassgold et al. (2004). The X-ray emission is modeled as a thermal plasma with a characteristic temperature $T_{x}$. The ionization rate scales as the inverse-square of the distance from the source according to (eq. [3.9] of S02):

$$
\zeta \approx \zeta_{\mathrm{x}}\left[\frac{r}{R_{\mathrm{x}}}\right]^{-2}\left[\frac{k T_{x}}{\epsilon_{\text {ion }}}\right] I_{p}\left(\tau_{\mathrm{x}}, \xi_{0}\right) \mathrm{s}^{-1} \text { (per H nucleus) }
$$

In the above, $R_{\mathrm{x}}$ fixes the height of the X-ray source above (or below) the disk center to mimic X-ray production in coronal loops. We use $\mathrm{R}_{\mathrm{x}}=4 \mathrm{R}_{*}=6.4 \mathrm{R}_{\odot}=4.45 \times 10^{11} \mathrm{~cm}$ as in previous studies (G97a, S02, SG13) but the results are not very sensitive to the adopted value of $\mathrm{R}_{\mathrm{x}}$ (IG99). The energy required to produce an ion pair is $\epsilon_{\text {ion }} \approx 37 \mathrm{eV}$. The term $I_{p}\left(\tau_{\mathrm{x}}, \xi_{0}\right)$ accounts for X-ray attenuation as a function of optical depth $\tau_{\mathrm{x}}$, where the latter depends on the photon energy $E$ and position in the disk. The term $\xi_{0}=\mathrm{E}_{0} / \mathrm{kT}_{\mathrm{x}}$ applies a low-energy cutoff $\mathrm{E}_{0}$ to the X-ray spectrum to account for wind absorption (IG99, S02). We use $\mathrm{E}_{0}=0.1 \mathrm{keV}$ here, as in previous work (S02, SG13). Figure 3 of SG13 shows the effect of decreasing the cutoff energy to $\mathrm{E}_{0}=0.01 \mathrm{keV}$.

The primary ionization rate $\zeta_{\mathrm{x}}$ is given by $(\mathrm{S} 02)$ :

$$
\zeta_{\mathrm{x}}=\frac{L_{x} \sigma\left(k T_{x}\right)}{4 \pi R_{x}^{2} k T_{x}}=1.13 \times 10^{-8}\left[\frac{L_{x}}{10^{30} \mathrm{erg} \mathrm{s}^{-1}}\right]\left[\frac{k T_{\mathrm{x}}}{\mathrm{keV}}\right]^{-(p+1)}\left[\frac{R_{\mathrm{x}}}{10^{12} \mathrm{~cm}}\right]^{-2} \mathrm{~s}^{-1}
$$

where $\sigma\left(k T_{x}\right)=\sigma(E)$ is the energy-dependent photoelectric X-ray absorption cross-section per $\mathrm{H}$ nucleus. It is evaluated using the expression $\sigma(E)=\sigma_{0}(\mathrm{E} / 1 \mathrm{keV})^{-p} \mathrm{~cm}^{-2}$ where $\sigma_{0}=$ 
$2.27 \times 10^{-22} \mathrm{~cm}^{2}$ and $p=2.485$ for solar-abundance disk plasma (G97a). Numerical values of $\zeta_{\mathrm{x}}$ for the cool and hot plasma components are given in Table 3 notes.

The X-ray optical depth used to compute the attenuation factor $I_{p}\left(\tau_{\mathbf{x}}, \xi_{0}\right)$ is

$$
\tau_{x}(r, z, E)=\left[\frac{r}{R_{\mathrm{x}}}\right] \sigma(\mathrm{E}) \mathrm{N}_{\perp, \operatorname{disk}}(r, z)
$$

where the vertically-integrated column density from infinity down to the height $\mathrm{z}$ above the disk midplane is

$$
\mathrm{N}_{\perp, \text { disk }}(r, z)=\int_{z}^{\infty} \mathrm{n}_{\mathrm{H}}(\mathrm{r}, \overline{\mathrm{z}}) \mathrm{d} \overline{\mathrm{z}} \quad \mathrm{cm}^{-2} .
$$

In general, $\tau_{x}$ will be smaller for the hot component at a given point in the disk, allowing the harder emission from the hot component to penetrate deeper into the disk. Note that for a given energy $E$ and height $z$, the optical depth $\tau_{x}$ is independent of $r$ as the result of a cancellation that occurs for the adopted surface density profile $\Sigma(r) \propto r^{-1}$. Also, it is worth emphasizing that the height above the midplane corresponding to $\tau_{x}=1$ depends sensitively on the adopted value of the gas surface density $\Sigma_{0}$ as shown in Figure 4 . At a given distance from the star, smaller adopted values of $\Sigma_{0}$ correspond to lower scale heights and deeper $\mathrm{X}$-ray penetration into the disk. The ionization rate for the cool and hot components as a function of $\tau_{x}$ is plotted in Figure 5.

The X-ray heating rate per unit volume is (G12)

$$
\Gamma_{\mathrm{x}}=\zeta \mathrm{n}_{\mathrm{H}} \mathrm{Q}
$$

where $Q$ is the heating rate per ionization. Several processes can affect the heating rate as discussed by G12. For predominantly molecular disk gas at $r \geq 1$ AU and the range of densities for the LkCa 15 inner disk (Table 3) we adopt $Q=17 \mathrm{eV}=2.72 \times 10^{-11}$ ergs, as in previous studies (SG13, G12). The X-ray heating rate then becomes

$$
\Gamma_{\mathrm{x}}=2.72 \times 10^{-11} \zeta n_{\mathrm{H}} \quad \operatorname{ergs~s}^{-1} \mathrm{~cm}^{-3}
$$

The heating rates at $r=1$ AU given in Table 3 can be scaled to other radii using the scaling relations for $\zeta$ and $\mathrm{n}_{\mathrm{H}}$ given in Table 3 notes. For the specific model adopted in this study the heating rate scales according to $\Gamma_{x} \propto r^{-4.25}$ as illustrated in Figure 6. As apparent from Figure 6 and Table 3, the X-ray heating is mostly due to the cool component as a 
result of its larger absorption cross-section and higher X-ray luminosity $\left(\mathrm{L}_{x, 1}\right)$. But for the disk model adopted here, X-ray ionization and heating are confined mainly to upper layers several scale heights above the midplane (Figs. 4 and 5). For the X-ray photon energies E $\lesssim$ $2.2 \mathrm{keV}$ that characterize the LkCa $15 X M M$-Newton spectrum, gas surface densities $\Sigma_{0}<$ $10^{-2} \mathrm{~g} \mathrm{~cm}^{-2}$ would be required in order for photons to penetrate to deeper layers within one scale height of the midplane (Fig. 4). Although such low surface densities were surmised in some early disk models of LkCa 15 (A11b), more recent ALMA observations (van der Marel et al. 2015) suggest higher gas densities $\Sigma_{0} \sim$ a few hundred $\mathrm{g} \mathrm{cm}^{-2}$ 
Table 3. X-ray Ionization and Heating Rates (LkCa 15)

\begin{tabular}{cccccccc}
\hline \hline $\begin{array}{c}\mathrm{r} \\
(\mathrm{AU})\end{array}$ & $\mathrm{z} / \mathrm{H}_{0}$ & $\begin{array}{c}\mathrm{E} \\
(\mathrm{keV})\end{array}$ & $\begin{array}{c}\Sigma_{0} \\
\left(\mathrm{~g} \mathrm{~cm}^{-2}\right)\end{array}$ & $\begin{array}{c}\mathrm{n}_{\mathrm{H}} \\
\left(\mathrm{cm}^{-3}\right)\end{array}$ & $\begin{array}{c}\mathrm{N}_{\perp} \\
\left(\mathrm{cm}^{-2}\right)\end{array}$ & $\begin{array}{c}\zeta \\
\left(\mathrm{s}^{-1}\right)\end{array}$ & $\begin{array}{c}\Gamma_{x} \\
\left(\mathrm{ergs} \mathrm{s}^{-1} \mathrm{~cm}^{-3}\right)\end{array}$ \\
\hline 1 & 5.0 & 0.4 & 100 & $9.80 \mathrm{e} 07$ & $1.35 \mathrm{e} 19$ & $2.46 \mathrm{e}-09$ & $6.56 \mathrm{e}-12$ \\
1 & 4.1 & 2.2 & 100 & $5.55 \mathrm{e} 09$ & $9.30 \mathrm{e} 20$ & $2.39 \mathrm{e}-11$ & $3.61 \mathrm{e}-12$ \\
\hline
\end{tabular}

Note. - The ionization rate $(\zeta)$ and heating rate $\left(\Gamma_{x}\right)$ for the cool $(\mathrm{E}=0.4 \mathrm{keV})$ and hot $(\mathrm{E}=2.2 \mathrm{keV})$ components are based on the inner disk model discussed in the text (Sec. 5.1.1). The rates are computed at $r=1 \mathrm{AU}$ for the specified value $z / \mathrm{H}_{0}$, which is the number of scale-heights above the midplane corresponding to unit X-ray optical depth $\left(\tau_{x}=1\right)$. At $r=1$ AU the disk scale height is $\mathrm{H}_{0} \equiv \mathrm{H}(\mathrm{r}=1 \mathrm{AU})=7.1 \times 10^{11} \mathrm{~cm}$ for an assumed disk midplane temperature $\mathrm{T}(r=1 \mathrm{AU})=400 \mathrm{~K}$. A disk gas surface density $\Sigma_{0} \equiv \Sigma(\mathrm{r}=1 \mathrm{AU})=100 \mathrm{~g} \mathrm{~cm}^{-2}$ is assumed, but the actual value is uncertain and modeldependent. The quantity $\mathrm{n}_{\mathrm{H}}$ is the number density of $\mathrm{H}$-nuclei at the specified point $(r, z)$ in the disk. The vertically-integrated H-nuclei column density from infinity down to the specified height above the midplane is $\mathrm{N}_{\perp}$ (eq. [4]). At $r=1 \mathrm{AU}$, values at the midplane $(z$ $=0)$ are $\mathrm{n}_{\mathrm{H}}(r=1 \mathrm{AU}, z=0)=2.38 \times 10^{13} \mathrm{~cm}^{-3}$ and $\mathrm{N}_{\perp}(r=1 \mathrm{AU}, z=0)=2.1 \times 10^{25} \mathrm{~cm}^{-2}$. The primary ionization rates (eq. [2]) for the cool and hot plasma components are $\zeta_{x, 1}=$ $2.53 \times 10^{-6} \mathrm{~s}^{-1}$ and $\zeta_{x, 2}=4.31 \times 10^{-9} \mathrm{~s}^{-1}$, respectively. These values are computed using the average $\mathrm{kT}$ and $\mathrm{L}_{x}$ values for the two models in Table 2 , namely $\overline{\mathrm{kT}_{1}}=0.4 \mathrm{keV}, \overline{\mathrm{kT}_{2}}$ $=2.2 \mathrm{keV}, \log \overline{\mathrm{L}_{\mathrm{x}, 1}}=30.26 \mathrm{ergs} \mathrm{s}^{-1}$, and $\log \overline{\mathrm{L}_{\mathrm{x}, 2}}=30.07 \mathrm{ergs} \mathrm{s}^{-1}$. The heating rate $\Gamma_{\mathrm{x}}$ $=\zeta \mathrm{n}_{\mathrm{H}} Q$ (eq. [5]) is computed using $\mathrm{Q}=17 \mathrm{eV}$. Scaling Relations: $\mathrm{H}(r) \propto r^{+1.5} \mathrm{~T}(r)$, or $\mathrm{H}(r) \propto r^{+1.25}$ for an assumed temperature dependence $\mathrm{T}(r)=400(r / 1 \mathrm{AU})^{-0.5}$. Scalings with radius are $\Sigma \propto r^{-1}, n_{\mathrm{H}} \propto r^{-2.25}, \zeta \propto r^{-2}, \Gamma_{x} \propto r^{-4.25}$. At a given radius, $n_{\mathrm{H}}(r, z)=$ $n_{\mathrm{H}}(r, z=0) \exp \left[-0.5(z / H(r))^{2}\right]$. 


\subsection{Comments on Circumstellar Disk Models}

Several different inner disk models have been proposed for LkCa 15 and the model adopted in our calculations above is only representative. We have assumed $\Sigma(r)=\Sigma_{0}(r / 1$ AU) $)^{q}$ with $q=-1$ and a gas surface density $\Sigma_{0}=10^{2} \mathrm{~g} \mathrm{~cm}^{-2}$. The adopted value of $\Sigma_{0}$ is similar to those determined in recent studies but is about an order-of-magnitude less than values derived in models of the minimum mass solar nebula (Weidenschilling 1977; Hayashi 1981). By way of comparison, Manara et al. (2014) found $\Sigma(r=1 \mathrm{AU})=83 \mathrm{~g} \mathrm{~cm}^{-2}$ for LkCa 15. Also, substitution of the LkCa 15 disk parameters determined in the ALMA study of van der Marel et al. (2015) into the surface density profile given in their eq. (1) yields $\Sigma(r$ $=1 \mathrm{AU})=286 \mathrm{~g} \mathrm{~cm}^{-2}$. This value includes their best-fit correction factor $\delta_{\text {gas }}=0.1$ which scales down the gas density in the inner disk $(1<r<45 \mathrm{AU})$ relative to the better-known gas distribution at larger radii. But van der Marel et al. note that the value of $\delta_{\text {gas }}$ is only constrained to within an order-of-magnitude. Several factors contribute to its uncertainty including inadequate spatial resolution to resolve gas in the inner disk, optical depth effects in the ${ }^{12} \mathrm{CO}$ line used to map the disk gas with ALMA, and possible asymmetries in the gas distribution. The much smaller surface density $\Sigma(r=1 \mathrm{AU})=10^{-3} \mathrm{~g} \mathrm{~cm}^{-2}$ obtained previously by A11b reflects differences in observational data and modeling strategy. The LkCa 15 disk model proposed in A11b was based on $880 \mu \mathrm{m}$ dust continuum observations obtained with the Submillimeter Array (SMA) and assumed an empty cavity devoid of dust and gas at radii $10 \leq r \leq 50 \mathrm{AU}$.

The value of the gas surface density power-law exponent $q$ is not well-constrained observationally in the inner disk but $q=-1$ is commonly used for LkCa 15 (e.g. Mulders et al. 2010; Manara et al. 2014; van der Marel et al. 2015). But other surface density profiles have been proposed such as the smooth viscous disk model of Isella et al. (2012) which uses a positive value $q=+2.15$ in the inner disk. In this model, the surface density increases in the inner disk and then starts to turn over at $r \sim 60$ AU to match the observationallyconstrained falloff in the outer disk (Fig. 3 of Isella et al. 2012). The smooth viscous model contrasts sharply with the discontinuous drop in $\Sigma(r)$ at $r \sim 50$ AU in the model of A11b. The above differences are relevant to X-ray ionization and heating calculations in the inner disk where the protoplanets are located since the radial dependence of the density of $\mathrm{H}$ nuclei is a function of the power-law index $q$ as $\mathrm{n}_{\mathrm{H}}(r) \propto r^{q-1.25}$ for an assumed temperature dependence $\mathrm{T}(r) \propto r^{-0.5}$.

We have adopted a disk temperature profile $\mathrm{T}(r)=\mathrm{T}_{0}(r / 1 \mathrm{AU})^{\beta} \mathrm{K}$ with the usual exponent $\beta=-0.5$ and normalized to $\mathrm{T}_{0}=400 \mathrm{~K}$ for a stellar temperature $\mathrm{T}_{\text {eff }}=4730 \mathrm{~K}$. Other studies have used $\mathrm{T}_{0}=100 \mathrm{~K}$ (A11a), $200 \mathrm{~K}$ (Manara et al. 2014), $250 \mathrm{~K}$ (Piétu et al. 2007, scaled to $r=1 \mathrm{AU}$ ), and $334 \mathrm{~K}$ (Bergin et al. 2004, scaled to $r=1 \mathrm{AU}$ and $\mathrm{T}_{\text {eff }}$ 
$=4730 \mathrm{~K})$. The temperature inferred from the ALMA study of van der Marel et al. (2015) is $\mathrm{T}_{0}=424 \mathrm{~K}$, which is scaled from the dust sublimation radius $\mathrm{T}(r=0.08 \mathrm{AU})=1500 \mathrm{~K}$ using $\beta=-0.5$. This latter value is nearly identical to that adopted here.

The adopted disk temperature profile enters into X-ray ionization and heating rate calculations through the scale height $\mathrm{H}(r) \propto \mathrm{T}(r)^{+0.5}$. This in turn affects the mass-density $\rho$ and $\mathrm{H}$-nuclei number density $\mathrm{n}_{\mathrm{H}}$ at the midplane (Sec. 5.1.1). At a given radius, a higher disk midplane temperature equates to a larger scale height $\mathrm{H}(r)$ and smaller $\mathrm{n}_{\mathrm{H}}$. But the dependence is weak and scales as $\mathrm{n}_{\mathrm{H}}(r) \propto \mathrm{T}(r)^{-0.5}$, so a factor of two uncertainty in $\mathrm{T}_{0}$ translates into only a factor of 1.4 uncertainty in $\mathrm{n}_{\mathrm{H}}$ at $r=1 \mathrm{AU}$.

\subsection{Comparison With Cosmic Ray Heating}

It is instructive to compare the X-ray heating rates in Table 3 with the rate expected from cosmic rays. The cosmic ray heating rate for a gas consisting of $\mathrm{H}$ and $\mathrm{H}_{2}$ is (Jonkheid et al. 2004)

$$
\Gamma_{c r}=\zeta_{c r}\left[2.5 \times 10^{-11} n_{\mathrm{H}_{2}}+5.5 \times 10^{-12} n_{\mathrm{H}}\right]\left(\mathrm{erg} \mathrm{cm}^{-3} \mathrm{~s}^{-1}\right)
$$

where the primary cosmic ray ionization rate in the interstellar medium (ISM) is $\zeta_{c r} \approx 5$ $\times 10^{-17} \mathrm{~s}^{-1}$. We assume that the LkCa 15 disk is predominantly molecular hydrogen and the second term in the above expression is negligible. At $r=1$ AU the molecular hydrogen density at scale heights corresponding to $\tau_{x}=1$ are half the $\mathrm{n}_{\mathrm{H}}$ values given in Table 3 , namely $\mathrm{n}_{\mathrm{H}_{2}}=4.9 \times 10^{7} \mathrm{~cm}^{-3}\left(z=5.0 \mathrm{H}_{0}, 0.4 \mathrm{keV}\right.$ photons $)$ and $2.8 \times 10^{9} \mathrm{~cm}^{-3}(z=4.1$ $\mathrm{H}_{0}, 2.2 \mathrm{keV}$ photons). We thus obtain $\Gamma_{c r}\left(r=1 \mathrm{AU}, z=5.0 \mathrm{H}_{0}\right)=6.12 \times 10^{-20} \mathrm{ergs} \mathrm{cm}^{-3} \mathrm{~s}^{-1}$ and $\Gamma_{c r}\left(r=1 \mathrm{AU}, z=4.1 \mathrm{H}_{0}\right)=3.48 \times 10^{-18} \mathrm{ergs} \mathrm{cm}^{-3} \mathrm{~s}^{-1}$. These values are $\sim 10^{6}-10^{8}$ times less than the X-ray heating rates. Thus, in the upper disk layers X-ray heating dominates and cosmic ray heating is negligible by comparison. But at the midplane, X-ray heating is negligible for the surface density assumed here $\left(\Sigma_{0}=10^{2} \mathrm{gm} \mathrm{cm}^{-2}\right)$ whereas cosmic ray

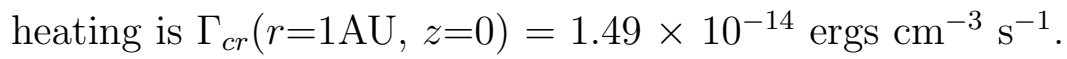

The winds and magnetic fields of $\mathrm{T}$ Tauri stars can impede penetration of cosmic rays into the protoplanetary disk, resulting in values of $\Gamma_{c r}$ that are less than the value appropriate for the ISM used above. The effects of T Tauri star winds and magnetic fields on cosmic rays were investigated by Cleeves et al. (2013) who concluded that reduced primary cosmic ray ionization rates of $\Gamma_{c r} \lesssim 10^{-18} \mathrm{~s}^{-1}$ are possible in protoplanetary disks, an order-ofmagnitude less than rates typically adopted for the ISM. The effect of such lower cosmic ray ionization rates would be to reduce cosmic ray heating near the disk midplane and strengthen 
the relative importance of X-ray heating in the upper disk layers.

\section{X-ray Irradiation of the Protoplanets and Circumplanetary Disks}

The analysis above has focused on X-ray irradiation of the LkCa 15 circumstellar disk but an assessment of X-ray effects on the atmospheres or circumplanetary disks of the protoplanets is also of interest. At present, little is known about the atmospheric properties of the $\mathrm{LkCa} 15$ protoplanets or their disks. But the detection of $\mathrm{H} \alpha$ emission from the innermost protoplanet at $r \approx 14.7 \mathrm{AU}$ by Sallum et al. (2015) does provide evidence that it is accreting and a circumplanetary disk is likely present. However, an attempt to detect $7 \mathrm{~mm}$ continuum emission using the VLA within the inner disk gap where the LkCa 15 protoplanets are located yielded negative results (Isella et al. 2014). Thus, crucial information on the temperature and surface density distribution of the planetary atmospheres and circumplanetary disks needed to calculate X-ray absorption and heating is lacking.

Nevertheless, we can show that if the LkCa 15 circumstellar disk surface density at a distance $r=1$ AU from the star is of order $\Sigma_{0} \sim 10^{2} \mathrm{~g} \mathrm{~cm}^{-2}$, as assumed in our calculations above, then little if any stellar X-ray emission reaches protoplanets that are near the midplane $(z \approx 0)$ at distances of $r \sim 15-20$ AU. At a distance $r$ from the star the intrinsic stellar X-ray flux $\mathrm{F}_{x}$ is reduced to $\mathrm{F}_{x} \mathrm{e}^{-\tau_{x}} / r^{2}$ where where $\tau_{x} \rightarrow \tau_{x}(r, z, \mathrm{E})$ is the energy-dependent X-ray optical depth through the circumstellar disk evaluated at the target point $(r, z)$. Assuming as before $\Sigma(r)=\Sigma_{0}(r / 1 \mathrm{AU})^{-1}$ and $\Sigma_{0}=10^{2} \mathrm{~g} \mathrm{~cm}^{-2}$ then we obtain by Equation (4)

$\mathrm{N}_{\perp, \text { disk }}(\mathrm{r}=15 \mathrm{AU}, \mathrm{z}=0)=1.4 \times 10^{24} \mathrm{~cm}^{-2}$. Using Equation (3) the optical depths for the cool and hot plasma components are computed to be $\tau_{x}(r=15 \mathrm{AU}, z=0, \mathrm{E}=0.4 \mathrm{keV})=1.6 \times$ $10^{6}$ and $\tau_{x}(r=15 \mathrm{AU}, z=0, \mathrm{E}=2.2 \mathrm{keV})=2.3 \times 10^{4}$. T Tauri stars may undergo intermittent bright X-ray flares during which plasma temperatures can briefly reach peak temperatures $\mathrm{T} \sim 100-250 \mathrm{MK}(\mathrm{E} \sim 9-22 \mathrm{keV})$ as has been well-documented in the Taurus Molecular Cloud (Franciosini et al. 2007) and Orion Nebula Cluster (Favata et al. 2005). No such high-temperature flares have been detected in LkCa 15 so far but optical depth calculations for the above range of peak values give $\tau_{x}(r=15 \mathrm{AU}, z=0, \mathrm{E}=9 \mathrm{keV})=685$ and $\tau_{x}(r=15$ $\mathrm{AU}, z=0, \mathrm{E}=22 \mathrm{keV})=74$. At these large optical optical depths it is obvious that any protoplanets located in or near the circumstellar disk midplane at $r \gtrsim 15 \mathrm{AU}$ will be shielded quite effectively from direct stellar X-ray emission.

The main uncertainty in the above $\tau_{x}$ calculations originates in the assumed surface density profile $\Sigma(r)=\Sigma_{0}(r / 1 \mathrm{AU})^{q}$. As already mentioned (Sec. 5.2), the normalization $\Sigma_{0}$ and power-law exponent $q$ are not well-constrained by observations for $\mathrm{LkCa} 15$, and the dependence of $\Sigma(r)$ with radius could be more complex than a simple power-law. But in 
order for stellar X-rays to penetrate through the circumstellar disk midplane to reach the protoplanets, much lower surface densities than assumed above would be required. Since $\tau_{x}$ $\propto \Sigma_{0}$ for fixed $(r, z, \mathrm{E})$, values $\Sigma_{0} \sim 10^{-3} \mathrm{~g} \mathrm{~cm}^{-2}$ (as in the model of A11b; see also SG13) would be needed to achieve $\tau_{x}(r=15 \mathrm{AU}, z=0, \mathrm{E}) \sim 1$. If the gas density is not currently that low it will eventually become so as the star-disk system evolves and the inner-disk gas dissipates.

\section{Summary}

A pointed observation of LkCa 15 with XMM-Newton confirms that it is a luminous $\mathrm{X}$-ray source, as previously inferred from a shorter Chandra observation. Although some low-level count-rate variability is likely present in the XMM-Newton X-ray light curves, no large-amplitude variability or flares were detected. The X-ray spectrum is characterized by a two-temperature thermal plasma with temperatures $\mathrm{kT}_{\text {cool }} \approx 0.4 \mathrm{keV}$ and a hotter component at $\mathrm{kT}_{\text {hot }} \approx 2.2 \mathrm{keV}$. The temperature of the hot component is about half that determined from the Chandra spectrum, suggesting that the hot component is variable as is often the case in T Tauri stars. Most of the X-ray ionization and heating of the circumstellar disk is due to the cool component. X-ray heating is restricted mainly to the upper disk layers assuming a disk gas surface density at $r=1 \mathrm{AU}$ of $\Sigma_{0} \sim 10^{2} \mathrm{~g} \mathrm{~cm}^{-2}$, as suggested by recent ALMA observations. Cosmic-ray heating is negligible compared to X-ray heating in the upper disk layers but few X-ray photons reach the disk midplane, where cosmic ray heating dominates. Unless the disk gas surface density is much less than the assumed value $\Sigma_{0} \sim$ $10^{2} \mathrm{~g} \mathrm{~cm}^{-2}$, little or no stellar X-ray emission is able to penetrate the circumstellar disk midplane to distances of $r \approx 15-20$ AU where the protoplanets are located.

This work was supported by NASA Astrophysics Data Analysis Program (ADAP) award NNX16AL71G. This work was based on observations obtained with XMM-Newton, an ESA science mission with instruments and contributions directly funded by ESA member states

and the USA (NASA). This research has made use of the HEASOFT data analysis software develeoped and maintained by HEASARC at NASA GSFC.

\section{REFERENCES}

Anders, E., \& Grevesse, N. 1989, Geochim. Cosmochim. Acta, 53, 197

Andrews, S.M., Rosenfeld, K.A., Wilner, D.J., \& Bremer, M. 2011b, ApJ, 742, L5 (A11a) 
Andrews, S.M., Wilner, D.J., Espaillat, C., et al. 2011b, ApJ, 732, 42 (A11b)

Balbus, S.A. \& Hawley, J.F. 1991, ApJ, 376, 214

Bergin, E., Calvet, N., Sitko, M.L. et al. 2004, ApJ, 614, L133

Cecchi-Pestellini, C., Ciaravella, A., \& Micela, A. 2006, A\&A, 458, L13

Cleeves, L.I., Adams, F.C., \& Bergin, E.A. 2013, ApJ, 772, 5

Drake, A.J., Graham, M.J., Djorgovski, S.G. et al. 2014, ApJS, 213, 9

Espaillat, C., Calvet, N., Luhman, K.L., Muzerolle, J., \& D’Alessio, P. 2008, ApJ, 682, L125

Espaillat, C., D’Alessio, P., Hernanández, J. et al. 2010, ApJ, 717, 441

Favata, F., Flaccomio, E., Reale, F. et al. 2005, ApJS, 160, 469

Franciosini, E., Pillitteri, I., Stelzer, B. et al. 2007, A\&A, 468, 485

Glassgold, A.E., Najita, J., \& Igea, J. 1997a, ApJ, 480, 344 (G97a)

Glassgold, A.E., Najita, J., \& Igea, J. 1997b, ApJ, 485, 920

Glassgold, A.E., Najita, J., \& Igea, J. 2004, ApJ, 615, 972

Gorenstein, P., 1975, ApJ, 198, 95

Güdel, M. et al. 2007, A\&A, 468, 353

Igea, J. \& Glassgold, A.E. 1999, ApJ, 518, 848 (IG99)

Hayashi, C. 1981, Supp. Progress Theor. Phys., 70, 35

Ireland, M.J. \& Kraus, A.L. 2014, in Exploring the Formation and Evolution of Planetary Systems, Proc. IAU Symposium No. 299, eds. M. Booth, B.C. Matthews, \& J.R. Graham (Cambridge U. Press), 199

Isella, A., Chandler, C.J., Carpenter, J.M., Pérez, L.M., \& Ricci, L. 2014, ApJ, 788, 129

Isella, A., Pérez, L.M., \& Carpenter, J.M. 2012, ApJ, 747, 136

Jonkheid, B., Faas, F.G.A., van Zadelhoff, G.-J., \& van Dischoeck, E.F. 2004, A\&A, 428, 511

Kenyon, S.J. \& Hartmann, L. 1995, ApJS, 101, 117 
Kraus, A.L. \& Hillenbrand, L.A. 2009, ApJ, 704, 531

Kraus, A.L. \& Ireland, M.J. 2012, ApJ, 745, 5 (KI12)

Manara, C.F., Testi, L., Natta, A., Rosotti, G., Benisty, M., Ercolano, B., \& Ricci, L. 2014, A\&A, 568, A18

Mulders, G.D., Dominik, C., \& Min, M. 2010, A\&A, 512, A11

Nguyen, D.C., Brandeker, A., van Kerkwijk, M.H., \& Jayawardhana, R.J. 2012, ApJ, 745, 119

Piétu, V., Dutrey, A., \& Guilloteau, S. 2007, A\&A, 467, 163

Sallum, S., Follette, K.B., Eisner, J.A. et al. 2015, Nature, 527, 342

Scelsi, L., Maggio, A., Micela, G., Briggs, K., \& Güdel, M. 2007, A\&A, 473, 589

Schmitt, J.H.M.M. \& Favata, F. 1999, Nature, 401, 44

Shang, H., Glassgold, A.E., Shu, F.H., \& Lizano, S. 2002, ApJ, 564, 853 (S02)

Simon, M., Dutrey, A., \& Guilloteau, S. 2000, ApJ, 545, 1034

Singh, K.P., Drake, S.A., \& White, N.E. 1996, AJ, 111, 2415

Skinner, S.L. \& Güdel, M. 2013, ApJ, 765, 3 (SG13)

Smith, R.K., Brickhouse, N.S., Liedahl, D.A., \& Raymond, J.C. 2001, ApJ, 556, L91

Strüder, L. et al., 2001, A\&A, 365, L18

Telleschi, A., Güdel, M., Briggs, K.R., Audard, M., \& Palla, F. 2007, A\&A, 468, 425

Thalmann, C., Mulders, G.D., Hodapp, K. et al. 2014, A\&A, 566, 51

Thalmann, C., Janson, M., Garufi, A. et al. 2016, ApJ, 828, L17

Torres, R.M., Loinard, L., Mioduszewski, A.J., \& Rodriguez, L.F., 2009, ApJ, 698, 242

Turner, M.J.L. et al., 2001, A\&A, 365, L27

van der Marel, N., van Dishoeck, E.F., Buderer, S., Pérez, L., \& Isella, A. 2015, A\&A, 579, A106 
Vuong, M.H., Montmerle, T., Grosso, N., Feigelson, E.D., Verstraete, L., \& Ozawa, H. 2003, A\&A, 408, 581

Weidenschilling, S.J. 1977, Ap\&SS, 51, 153 


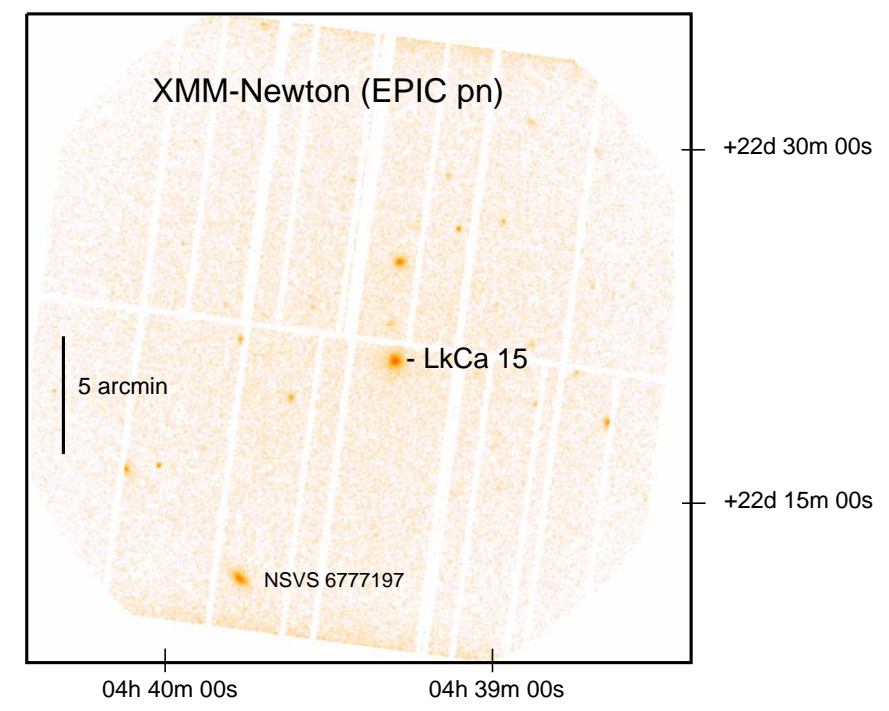

Fig. 1.- Broad-band (0.2 - $8 \mathrm{keV})$ lightly-smoothed EPIC pn image of LkCa 15 and surrounding region. The image has been time-filtered to remove intervals affected by background flares. The Algol-type binary NSVS 6777196 is marked near the south edge of the pn fieldof-view. The bright source $4.2^{\prime}$ north of LkCa 15 is classified as non-stellar in the HST v. 2.3 Guide Star Catalog (GSC J043916.97+222515.59). The image is displayed on a log intensity scale with N up and E to left. The coordinates are equinox J2000. 


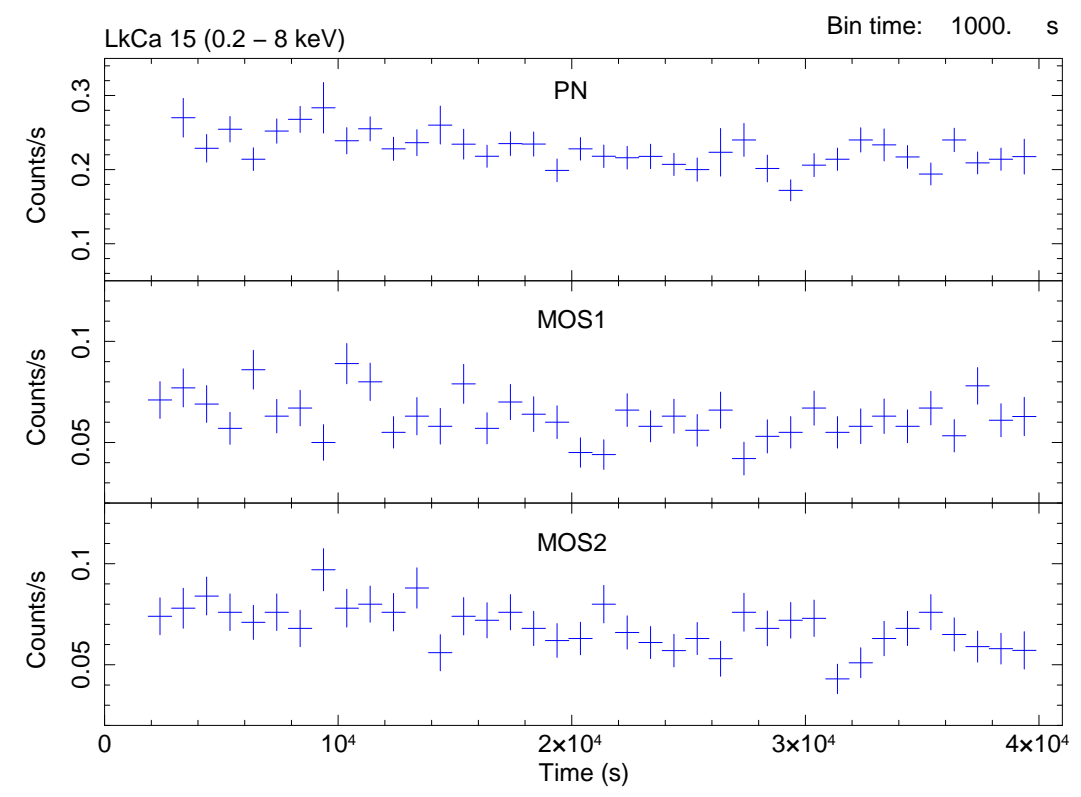

Fig. 2.- Background-subtracted EPIC light curves of LkCa 15 in the $0.2-8 \mathrm{keV}$ range extracted from a circular region of radius $20^{\prime \prime}$ centered on the source. Binned at $1000 \mathrm{~s}$ intervals. Intervals of high background have been removed. Times are relative to 19:00 UTC on 2014 Feb. 20. 

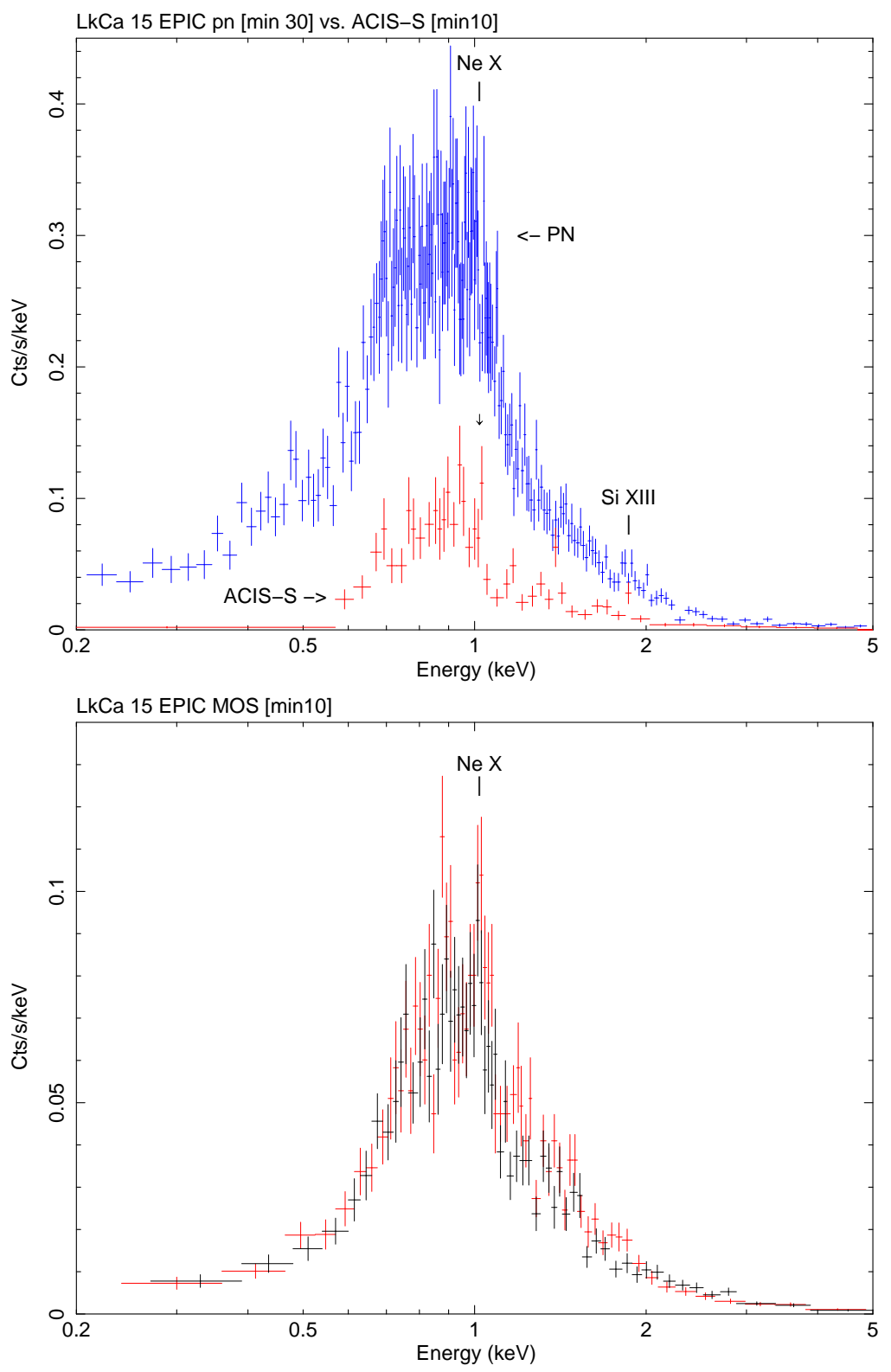

Fig. 3.- Background-subtracted X-ray spectra of LkCa 15 obtained with XMM-Newton EPIC (ObsId 0722340101) and Chandra ACIS-S (ObsId 10999). Intervals of high-background emission have been excluded from the EPIC spectra. ACIS-S background is negligible. Top: EPIC pn (blue, 7568 net counts, binned to a minimum of 30 counts per bin) overlaid with ACIS-S (red, 590 net counts, binned to a minimum of 10 counts per bin). Possible Ne $\mathrm{X}(1.02 \mathrm{keV})$ and Si XIII $(1.86 \mathrm{keV})$ lines are marked. Bottom: Overlay of EPIC MOS1 (black; 2463 net counts) and MOS2 (red; 2743 net counts) spectra, binned to a minimum of 10 counts per bin. A spectral feature visible in both MOS1 and MOS2 identified as Ne X $(1.02 \mathrm{keV})$ is marked. 


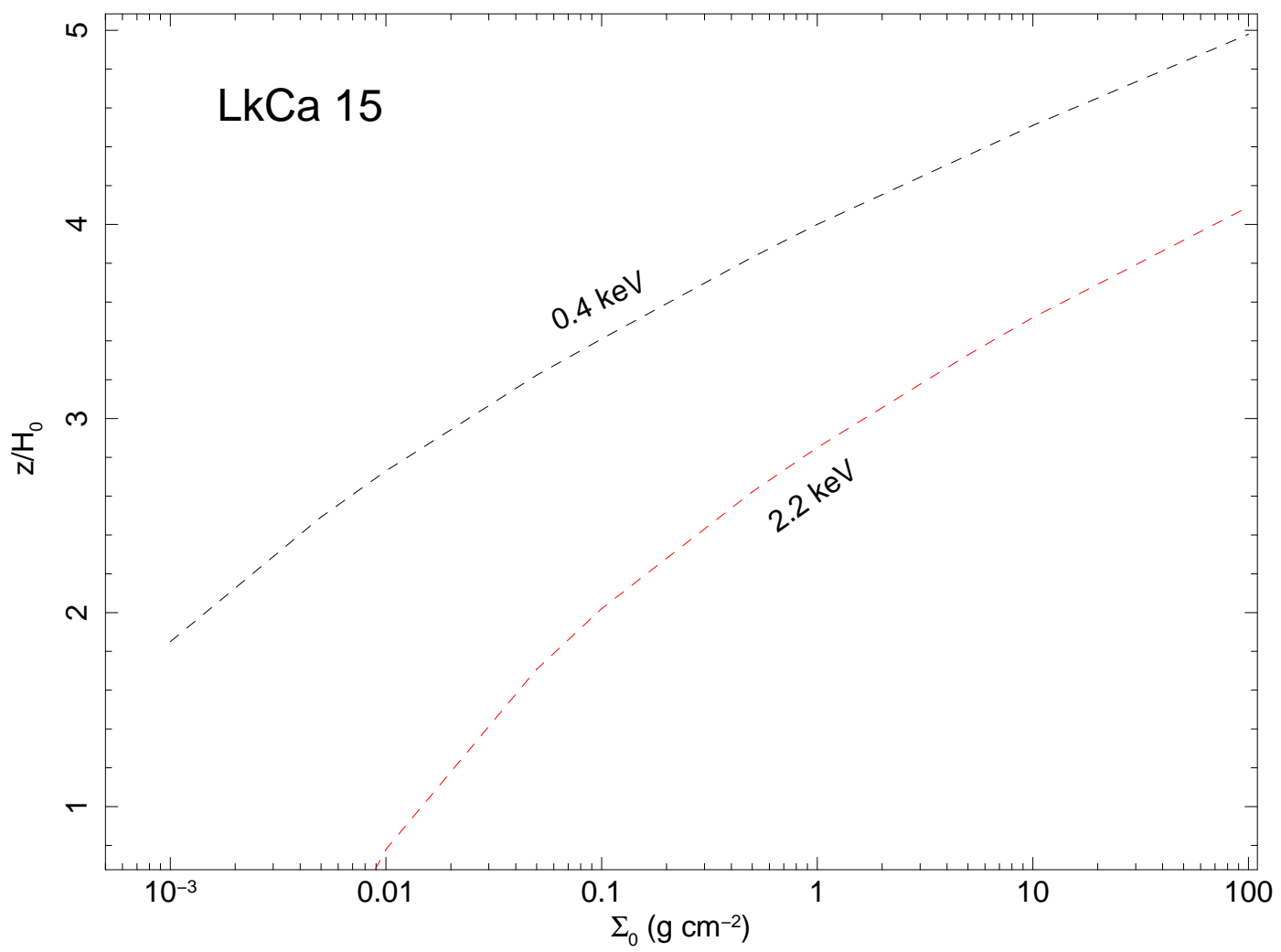

Fig. 4.- Distance above the disk midplane corresponding to unit X-ray optical depth $\left(\tau_{x}=\right.$ 1) for the cool and hot plasma components as a function of the disk gas surface density $\Sigma_{0}$ at $r=1 \mathrm{AU}$. The height above the midplane is expressed in units of the scale height $\mathrm{H}_{0} \equiv$ $\mathrm{H}(r=1 \mathrm{AU})=7.1 \times 10^{11} \mathrm{~cm}$ for an assumed disk midplane temperature $\mathrm{T}(r=1 \mathrm{AU})=$ $400 \mathrm{~K}$. Very low surface densities $\Sigma_{0}<0.01 \mathrm{~g} \mathrm{~cm}^{-2}$ are required for the X-rays to penetrate to depths below one scale height. 


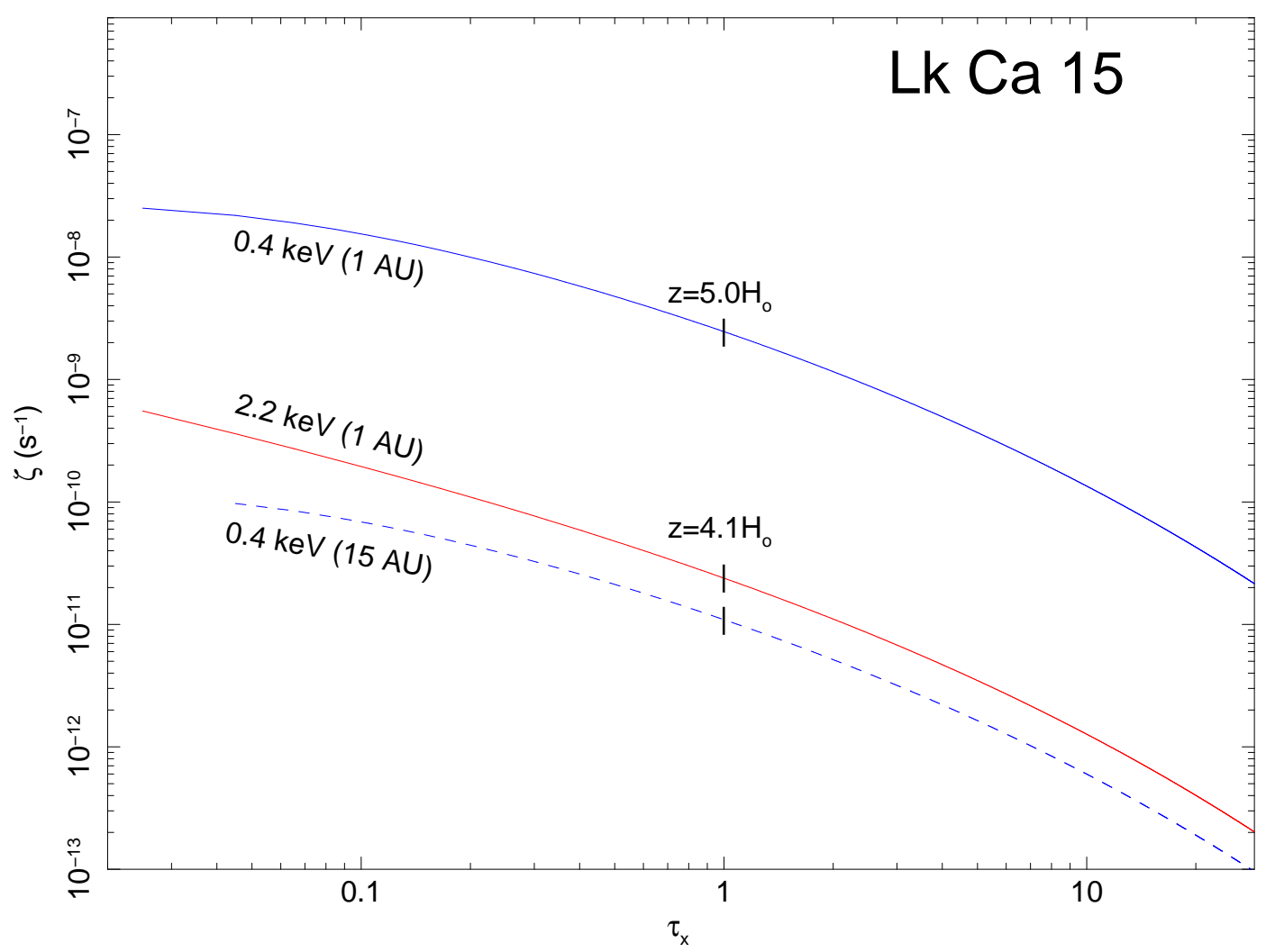

Fig. 5.- X-ray ionization rate versus X-ray optical depth for the LkCa 15 disk as computed for the model adopted in this study (Sec. 5; Table 3). The two solid curves show the ionization rates for the cool and hot components evaluated at a radial distance $\mathrm{r}=1 \mathrm{AU}$ from the star. The dashed curve shows the rate for the cool component at $\mathrm{r}=15 \mathrm{AU}$. The short vertical lines mark the scale height corresponding to $\tau_{x}=1$ assuming a disk gas surface density $\Sigma_{0} \equiv \Sigma(r=1 \mathrm{AU})=10^{2} \mathrm{~g} \mathrm{~cm}^{-2}$. At $r=1 \mathrm{AU}$ the scale height is $\mathrm{H}_{0}=7.1 \times 10^{11}$ $\mathrm{cm}(=0.048 \mathrm{AU})$ for an assumed disk temperature $\mathrm{T}(r=1 \mathrm{AU})=400 \mathrm{~K}$. The ionization rate for a specific height $z$ scales as $\zeta(r) \propto r^{-2}$. 


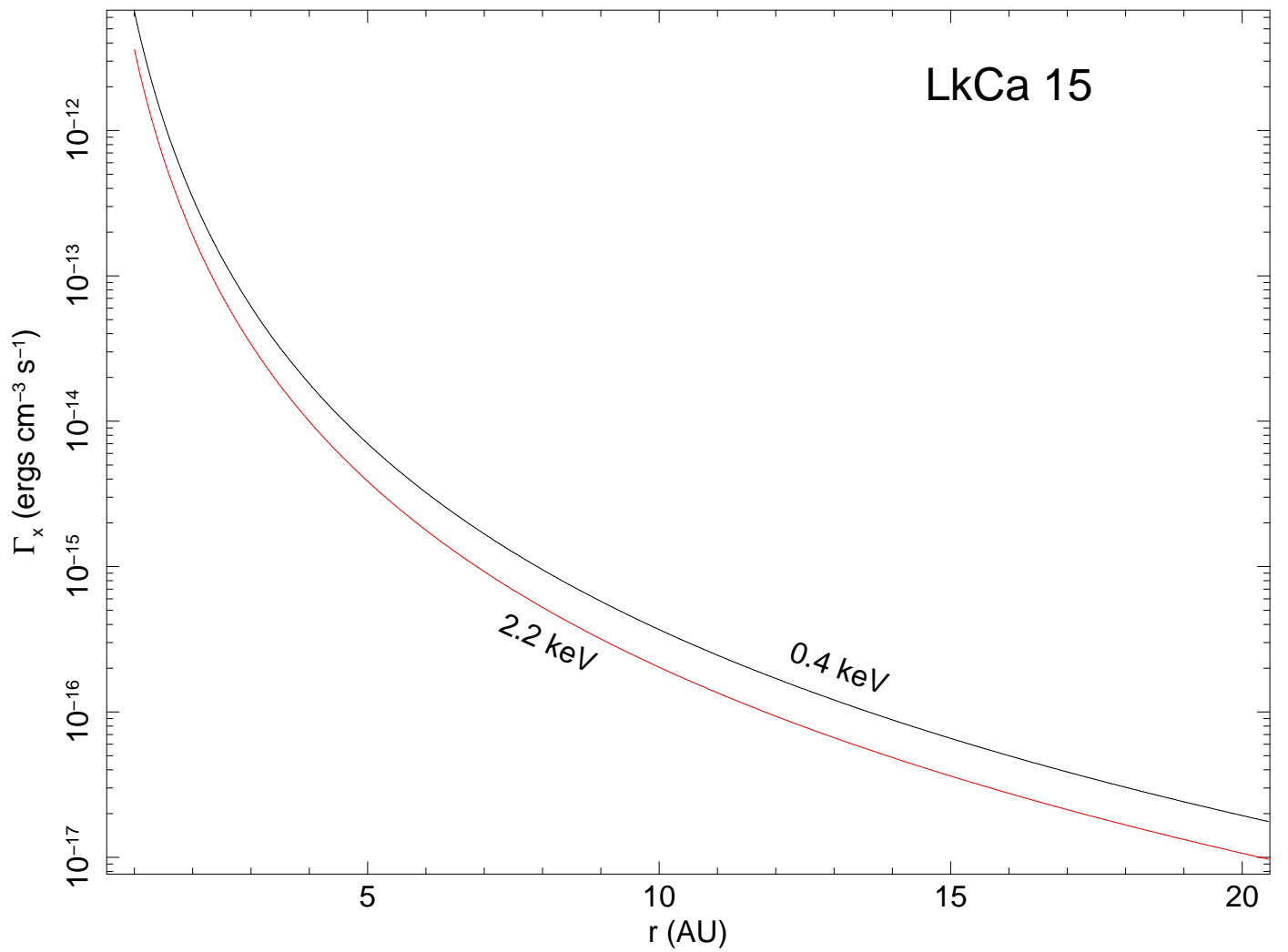

Fig. 6.- Disk heating rate per unit volume as a function of radial distance from the star for the cool and hot plasma components. The heating rate is evaluated at the distance above the midplane corresponding to $\tau_{x}=1$ (see Fig. 4), which is $\mathrm{z} / \mathrm{H}_{0}=5.0(0.4 \mathrm{keV})$ and $\mathrm{z} / \mathrm{H}_{0}$ $=4.1(2.2 \mathrm{keV})$. For the disk model adopted in this study $\Gamma_{x} \propto r^{-4.25}$. 